\title{
On the Recognition of Fan-Planar and Maximal Outer-Fan-Planar Graphs *
}

\author{
M. A. Bekos ${ }^{1}$, S. Cornelsen ${ }^{2}$, L. Grilli ${ }^{3}$, S.-H. Hong ${ }^{4}$, M. Kaufmann ${ }^{1}$ \\ 1 Wilhelm-Schickard-Institut für Informatik, Universität Tübingen, Germany \\ \{bekos,mk\}@informatik.uni-tuebingen.de \\ 2 Dept. of Computer and Information Science, University of Konstanz, Germany \\ sabine.cornelsen@uni-konstanz.de \\ 3 Dipartimento di Ingegneria, Università degli Studi di Perugia, Italy \\ luca.grilli@unipg.it \\ 4 School of Information Technologies, University of Sydney, Australia \\ shhong@it.usyd.edu.au
}

\begin{abstract}
Fan-planar graphs were recently introduced as a generalization of 1-planar graphs. A graph is fan-planar if it can be embedded in the plane, such that each edge that is crossed more than once, is crossed by a bundle of two or more edges incident to a common vertex. A graph is outer-fan-planar if it has a fan-planar embedding in which every vertex is on the outer face. If, in addition, the insertion of an edge destroys its outer-fan-planarity, then it is maximal outer-fan-planar.

In this paper, we present a polynomial-time algorithm to test whether a given graph is maximal outerfan-planar. The algorithm can also be employed to produce an outer-fan-planar embedding, if one exists. On the negative side, we show that testing fan-planarity of a graph is NP-hard, for the case where the rotation system (i.e., the cyclic order of the edges around each vertex) is given.
\end{abstract}

\section{Introduction}

A simple drawing of a graph is a representation of a graph in the plane, where each vertex is represented by a point and each edge is a Jordan curve connecting its end-points such that no edge contains a vertex in its interior, no two edges incident to a common end-vertex cross, no edge crosses itself, no two edges meet tangentially, and no two edges cross more than once.

An important subclass of drawn graphs is the class of planar graphs, in which there exist no crossings between edges. Although planarity is one of the most desirable properties when drawing a graph, many real-world graphs are in fact non-planar.

On the other hand, it is widely accepted that edge crossings have negative impact on the human understanding of a graph drawing [26] and simultaneously it is NP-complete in general to find drawings with minimum number of edge crossings [16. This motivated the study of "almost planar" graphs which may contain crossings as long as they do not violate some prescribed forbidden crossing patterns. Typical examples of such graphs include $k$-planar graphs [27, $k$-quasi planar graphs [2, RAC graphs [9] and fan-crossing free graphs [7.

Fan-planar graphs were recently introduced in the same context [21. Typically, a fan-planar drawing of graph $G=(V, E)$ is a simple drawing which allows for more than one crossing on an edge $e \in E$ if and only if the edges that cross $e$ are incident to a common vertex on the same side of $e$. Such a crossing is called fan-crossing (see Fig 1a). An equivalent definition can be stated by means of forbidden crossing patterns;

* This work started at the Bertinoro Workshop on Graph Drawing 2014. We thank the organizers and the participants of the workshop for the useful discussions on this topic. The work of M.A. Bekos is implemented within the framework of the Action "Supporting Postdoctoral Researchers" of the Operational Program "Education and Lifelong Learning" (Action's Beneficiary: General Secretariat for Research and Technology), and is co-financed by the European Social Fund (ESF) and the Greek State. L. Grilli was partly supported by the MIUR project AMANDA "Algorithmics for MAssive and Networked DAta", prot. 2012C4E3KT_001. S. Hong was partly supported by her ARC Future Fellowship and Humboldt Fellowship. 


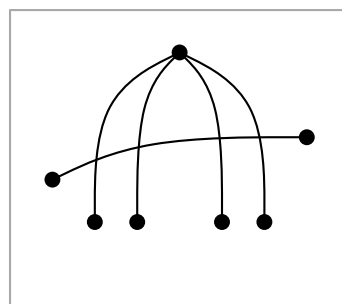

(a) Fan-crossing

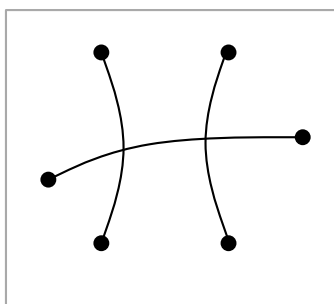

(b) Forbidden pattern I

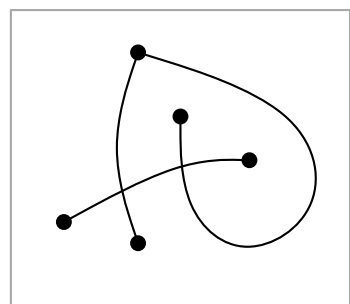

(c) Forbidden pattern II

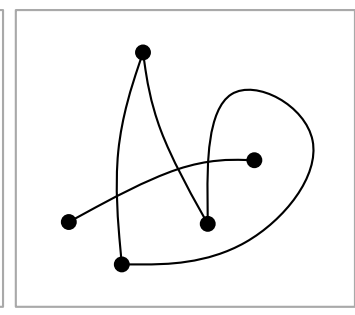

(d) Triangle crossing

Fig. 1. (taken from [21])(a) Illustration of a fan-crossing. (b) Forbidden crossing pattern I: An edge cannot be crossed by two independent edges. (c) Forbidden crossing pattern II: An edge cannot be crossed by two edges having their common end-point on different sides of it. (d) Forbidden crossing pattern II implies that an edge cannot be crossed by three edges forming a triangle.

see Fig. 1b, 1c and 1d, A graph is fan-planar if it admits a fan-planar drawing. Note that the class of fanplanar graphs is in a sense the complement of the class of fan-crossing free graphs [7, which simply forbid fan-crossings.

To the best of our knowledge the only known result for this particular class of graphs is due to Kaufmann and Ueckerdt [21, who showed that a fan-planar graph on $n$ vertices cannot have more than $5 n-10$ edges and this bound is tight. An outer-fan-planar drawing is a fan-planar drawing in which all vertices are on the outer face. A graph is outer-fan-planar if it admits an outer-fan-planar drawing. An outer-fan-planar graph is maximal outer-fan-planar if adding any edge to it yields a graph that is not outer-fan-planar. Note that the forbidden pattern II is irrelevant for outer-fan-planarity. Our main contribution is a polynomial time algorithm for the recognition of maximal outer-fan-planar graphs and significant insights in their structural properties (see Section 2). We also prove that the general fan-planar problem is NP-hard, for the case where the rotation system (i.e., the circular order of the edges around each vertex) is given (see Section 3). The question how to test (non-maximal) outer-fan-planarity efficiently is left open.

\subsection{Related Work}

As already stated, $k$-planar graphs [27, $k$-quasi planar graphs [2], RAC graphs [9] and fan-crossing free graphs [7] are closely related to the class of graphs we study. A graph is $k$-planar, if it can be embedded in the plane with at most $k$ crossings per edge. Obviously, 1-planar graphs are also fan-planar. A 1-planar graph with $n$ vertices has at most $4 n-8$ edges and this bound is tight [5]1325. Grigoriev and Bodlaender [17], and, independently Kohrzik and Mohar 22 proved that the problem of determining whether a graph is 1-planar is NP-hard and remains NP-hard, even if the deletion of an edge makes the input graph planar 6 .

On the positive side, Eades et al. [10] presented a linear time algorithm for testing maximal 1-planarity of graphs with a given rotation system. Testing outer-1-planarity of a graph can be solved in linear time, as shown independently by Auer et al. 4] and Hong et al. [19]. It is worth to note that an outer-1-planar graph is always planar [4, while this is not true in general for outer-fan-planar graphs. Indeed, the complete graph $K_{5}$ is outer-fan-planar, but not planar.

The well-known Fary's theorem [14] proved that every plane graph admits a straight-line drawing. However, Thomassen [28] presented two forbidden subgraphs for straight-line drawings of 1-plane graphs. Hong et al. 20] gave a linear-time testing and drawing algorithm to construct a straight-line 1-planar drawing, if it exists. Recently, Nagamochi solved the more general problem of straight-line drawability for wider classes of embedded graphs [23. On the other hand, Eggleton showed that every outer-1-planar graph admits an outer-1-planar straight-line drawing [12].

A drawn graph is called $k$-quasi planar if it does not contain $k$ mutually crossing edges. Fan-planar graphs are 3-quasi planar, since they cannot contain three independent edges that mutually cross. It is conjectured that the number of edges of a $k$-quasi planar graph is linear in the number of its vertices. Pach et al. 24] 
and Ackerman [1] showed that this conjecture holds for 3- and 4-quasi planar graphs, respectively. Fox and Pach [15] showed that every $k$-quasi-planar graph with $n$ vertices has at most $O\left(n \log ^{1+o(1)} n\right)$ edges.

A different forbidden crossing pattern arises in RAC drawings where two edges are allowed to cross, as long as the crossings edges form right angles. Graphs that admit such drawings (with straight-line edges) are called right-angle crossing graphs or RAC graphs, for short. Didimo et al. [9] showed that a RAC graph with $n$ vertices cannot have more than $4 n-10$ edges and that this bound is tight. It is also known that a RAC graph is quasi planar [9], while a maximally dense RAC graph (i.e., a RAC graph with $n$ vertices and exactly $4 n-10$ edges) is 1-planar [11. Testing whether a given graph is a RAC graph is NP-hard [3]. Dekhordi and Eades 8 , proved that every outer-1-plane graph has a straight-line RAC drawing, at the cost of exponential area.

\subsection{Preliminaries}

We consider finite, undirected and simple graphs. A drawing of a graph maps vertices to points in the plane and edges to simple closed curves between the points corresponding to the end-vertices of the edge. For a given drawing, we say that two edges cross if the interiors of their corresponding curves share a common point. A drawing is simple if no edge contains a vertex in its interior, no two edges incident to a common end-vertex cross, no edge crosses itself, no two edges meet tangentially, and no two edges cross more than once. The rotation system of a drawing is the counterclockwise order of the incident edges around each vertex. The embedding of a drawn graph consists of its rotation system and for each edge the sequence of edges crossing it. For a graph $G$ and a vertex $v \in V[G]$, we denote by $G-\{v\}$ the graph that results from $G$ by removing $v$. A fan-planar drawing of a graph $G=(V, E)$ is a simple drawing such that, for each edge $e \in E$, the edges that cross $e$, if any, are all incident to a common vertex $v$ on the same side of $e$. Such a crossing is called a fan-crossing. An equivalent definition can be stated by means of forbidden crossing patterns; see Fig. 1b, 1c and 1d. A graph is fan-planar if it admits a fan-planar drawing. An drawing is outer-fan-planar if it is a fan-planar drawing with all vertices on the outer face. A graph is outer-fan-planar if it admits an outer-fan-planar drawing. An outer-fan-planar graph is maximal outer-fan-planar if adding any edge to it yields a graph that is not outer-fan-planar. Note that forbidden pattern II is irrelevant for outer-fan-planarity.

We now briefly recall the SPQR-tree data structure [18. Two vertices $v$ and $w$ are a separation pair of a connected graph $G$ if the graph that results from $G$ by deleting $v$ and $w$ is not connected. A graph is 3-connected if it contains more than three vertices but no separation pair. An SPQR-tree is a labeled tree that represents the decomposition of a biconnected graph into 3-connected components. Each node $x$ of an SPQR-tree is labeled with a multi-graph $G_{x}$ - called the skeleton of $x$. There are four different types of labels with the following skeletons: (i) $S$-nodes: a simple cycle. (ii) $P$-nodes: three or more parallel edges. (iii) $R$-nodes: a simple 3 -connected graph. (iv) $Q$-nodes: a single edge. No two $S$-nodes, nor two $P$-nodes are adjacent in an SPQR-tree. For each node $x$ of an SPQR-tree there is a one-to-one correspondence of the edges of the skeleton of $G_{x}$ and the edges incident to $x$. Further, let $\{x, y\}$ be an edge of an SPQR-tree and let $e_{x}$ and $e_{y}$ be the edges of $G_{x}$ and $G_{y}$, respectively, that are assigned to $\{x, y\}$. Then, $e_{x}$ and $e_{y}$ have the same end-vertices - say $u$ and $v$. Moreover, let $x^{\prime}$ and $y^{\prime}$ be two nodes in different connected components of $T$ without the edge $\{x, y\}$. Then $G_{x^{\prime}}$ and $G_{y^{\prime}}$ share at most $u$ and $v$ as common vertices.

An SPQR-tree represents the (multi-)graph constructed by iteratively merging edges of the SPQR-tree as follows. For an edge $\{x, y\}$ of the current tree, let $G_{x}$ and $G_{y}$ be the graphs currently associated with $x$ and $y$, respectively. Remove the edge associated with $\{x, y\}$ from both $G_{x}$ and $G_{y}$ - except if they are $Q$-nodes. Let the graph associated with the node that results from merging $x$ and $y$ be the union of (the remaining parts of) $G_{x}$ and $G_{y}$.

The edges of a skeleton are called virtual edges if they correspond to a tree edge that is not incident to a $Q$-node and real edges otherwise. Note that real edges correspond to the edges of the graph represented by the SPQR-tree. Every biconnected graph has a unique SPQR-tree and the SPQR-tree of a biconnected graph can be constructed in linear time [18. 


\section{Recognizing and Drawing Maximal Outer-Fan-Planar Graphs}

In this section, we prove that given a graph $G=(V, E)$ on $n$ vertices, there is a polynomial time algorithm to decide whether $G$ is maximal outer-fan-planar and if so a corresponding straight-line drawing can be computed in linear time. We first observe that biconnectivity is a necessary condition for maximal outer-fanplanarity. In other words, a simply connected graph that is not biconnected cannot be maximal outer-fanplanar. Indeed, if an outer-fan-planar drawing has a cut-vertex $c$, it is easy to see that it is always possible to draw an edge connecting two neighbors of $c$ while preserving the outer-fan-planarity. Further, because of the following lemma, we only have to check whether $G$ admits a straight-line fan-planar drawing on a circle $\mathcal{C}$; such a drawing is completely determined by the cyclic ordering of the vertices on $\mathcal{C}$.

Lemma 1. A biconnected graph $G$ is outer-fan-planar if and only if it admits a straight-line outer-fan-planar drawing in which the vertices of $G$ are restricted on a circle $\mathcal{C}$.

Proof. Let $G$ be an outer-fan-planar graph and let $\Gamma$ be an outer-fan-planar drawing of $G$. We will only show that $G$ has a straight-line outer-fan-planar drawing whose vertices lie on a circle $\mathcal{C}$ (the other direction is trivial). The order of the vertices along the outer face of $\Gamma$ completely determines whether two edges cross, as in a simple drawing no two incident edges can cross and any two edges can cross at most once. Now, assume that two edges cross another edge in $\Gamma$. Then, both edges have to be incident to the same vertex; hence, cannot cross each other. So, the order of the crossings on an edge is also determined by the order of the vertices on the outer face. Therefore, we can construct a drawing $\Gamma_{\mathcal{C}}$ by placing the vertices of $G$ on a circle $\mathcal{C}$ preserving their order in the outer face of $\Gamma$ and draw the edges as straight-line segments.

Since fan-planar graphs with $n$ vertices have at most $5 n-10$ edges [21, we may assume that the number of edges is linear in the number of vertices. We first consider the case that $G$ is 3-connected (see Section 2.1) and then using SPQR-trees we show how the problem can be solved for biconnected graphs (see Section 2.2).

\subsection{The 3-Connected Case}

Assume that a straight-line drawing of a 3-connected graph $G$ with $n$ vertices on a circle $\mathcal{C}$ is given. Let $v_{1}, \ldots, v_{n}$ be the order of the vertices around $\mathcal{C}$. An edge $\left\{v_{i}, v_{j}\right\}$ is an outer edge, if $i-j \equiv \pm 1(\bmod n)$, a 2-hop, if $i-j \equiv \pm 2(\bmod n)$, and a long edge otherwise. $G$ is a complete 2-hop graph, if there are all outer edges and all 2-hops, but no long edges. Two crossing long edges are a scissor if their end-points form two consecutive pairs of vertices on $\mathcal{C}$. We say that a triangle is an outer triangle if two of its three edges are outer edges. We call an outer-fan-planar drawing maximal, if adding any edge to it yields a drawing that is not outer-fan-planar.

Our algorithm is based on the observation that if a graph is 3-connected maximal outer-fan-planar, then it is a complete 2-hop graph, or we can repeatedly remove any degree-3 vertex from any 4-clique until only a triangle is left. In a second step, we reinsert the vertices maintaining outer-fan-planarity (if possible). It turns out that we have to check a constant number of possible embeddings. In the following, we prove some necessary properties. The first three lemmas are used in the proof of Lemma 5. Their proofs are based on the 3 -connectivity of the input graph; see also Fig. 2 , 3 and $2 \mathrm{c}$

Lemma 2. Let $G$ be a 3-connected outer-fan-planar graph embedded on a circle $\mathcal{C}$. If two long edges cross, then two of its end-points are consecutive on $\mathcal{C}$.

Proof. Assume to the contrary that there exist two long crossing edges $\left\{v_{i_{1}}, v_{i_{3}}\right\}$ and $\left\{v_{i_{2}}, v_{i_{4}}\right\}$, such that $2 \leq i_{1} \leq i_{2}-2 \leq i_{3}-4 \leq i_{4}-6$ and $i_{4}=n$; see Fig 2 . Since $G$ is 3 -connected, there has to be a vertex $v_{j_{1}}$ with $i_{1}<j_{1}<i_{2}$, such that $v_{j_{1}}$ is adjacent to a vertex not in $\left\{v_{i_{1}}, \ldots, v_{i_{2}}\right\}$. By outer-fan-planarity, this can only be $v_{i_{3}}$ or $v_{i_{4}}$; say without loss of generality $v_{i_{3}}$. Likewise there is a vertex $v_{i_{2}}$ with $i_{2}<j_{2}<i_{3}$, such that $v_{j_{2}}$ is adjacent to a vertex not in $\left\{v_{i_{2}}, \ldots, v_{i_{3}}\right\}$. By outer-fan-planarity this can now only be $v_{i_{4}}$. But now outer-fan-planarity does not permit to add an edge connecting the two parts separated by $v_{i_{3}}$ and $v_{i_{4}}$. 


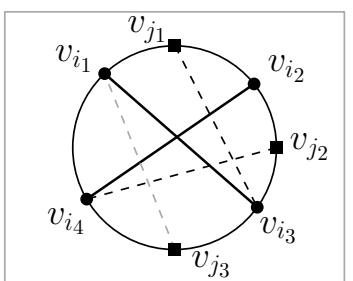

(a)

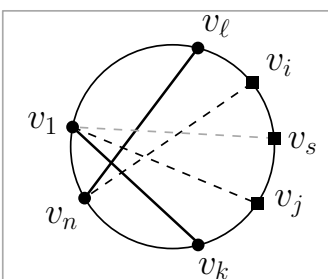

(b)

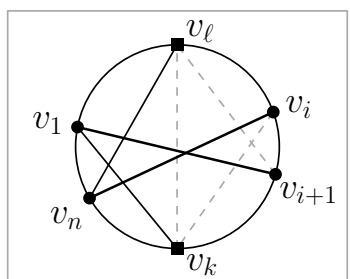

(c)

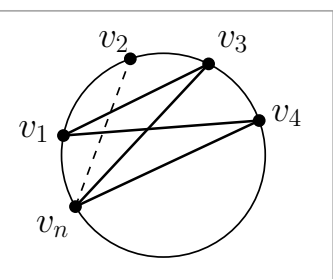

(d)

Fig. 2. Different configurations used in: (a) Lemma 2, (b) Lemma 3 , (c, d) Lemma 4.

Lemma 3. Let $G$ be a 3-connected outer-fan-planar graph embedded on a circle $\mathcal{C}$. If there are two long crossing edges, then there is a scissor, as well.

Proof. Let $e$ and $e^{\prime}$ be two long crossing edges. By Lemma 2 it follows that two of the end-points of $e$ and $e^{\prime}$ are consecutive on $\mathcal{C}$. So, assume without loss of generality that the vertices on $\mathcal{C}$ are labeled such that $e=\left\{v_{1}, v_{k}\right\}$ and $e^{\prime}=\left\{v_{\ell}, v_{n}\right\}$ for some $\ell<k$; see Fig 3 . If $k=\ell+1$, then the lemma holds. If this is not the case, then among all crossing long edges with end-vertices $v_{1}$ and $v_{n}$ on one hand and end-vertices between $v_{\ell}$ and $v_{k}$ on the other hand, let edges $\left\{v_{1}, v_{j}\right\}$ and $\left\{v_{n}, v_{i}\right\}$, with $\ell \leq i<j \leq k$ be the ones for which the difference $j-i$ is minimal. Obviously, if $j=i+1$, then the edges $\left\{v_{1}, v_{j}\right\}$ and $\left\{v_{n}, v_{i}\right\}$ are a scissor. Assume now that $j>i+1$. Since $v_{i}$ and $v_{j}$ cannot be a separation pair, there has to be an edge between a vertex $v_{s}$ with $j<s<i$ and a vertex $v_{t}$ with $t<i$ or $t>j$. By outer-fan-planarity $t=1$ or $t=n$. This contradicts the choice of $\left\{v_{1}, v_{j}\right\}$ and $\left\{v_{i}, v_{n}\right\}$.

Lemma 4. Let $G$ be a 3-connected graph embedded on a circle $\mathcal{C}$ with a maximal outer-fan-planar drawing. If $G$ contains a scissor, then its end-vertices induce a $K_{4}$.

Proof. Assume without loss of generality that the vertices on $\mathcal{C}$ are labeled such that $\left\{v_{1}, v_{i+1}\right\}$ and $\left\{v_{i}, v_{n}\right\}$ is a scissor, for some $1<i<n$. We have to show that $\left\{v_{1}, v_{i}\right\} \in E[G]$ and $\left\{v_{n}, v_{i+1}\right\} \in E[G]$. By outerfan-planarity there cannot be an edge $\left\{v_{\ell}, v_{k}\right\}$, such that $1<\ell<i$ and $i+1<k<n$; see Fig. 2c. Since $v_{1}$ and $v_{i}$ cannot be a separation pair, there has to be an edge between $v_{n}$ or $v_{i+1}$ and a vertex $v_{\ell}$ with $1<\ell<i$; say from $v_{n}$. Similarly, since $v_{n}$ and $v_{i+1}$ cannot be a separation pair, there has to be an edge between $v_{1}$ or $v_{i}$ and a vertex $v_{k}$, with $i+1<k<n$. By outer-fan-planarity, this can only be an edge from $v_{1}$, as otherwise edge $\left\{v_{1}, v_{i+1}\right\}$ would be crossed by two independent edges; see Fig. $2 \mathrm{c}$. As a consequence, there cannot be an edge between $v_{i}$ and a vertex $v_{k}, i+1<k<n$ nor an edge between $v_{i+1}$ and a vertex $v_{\ell}, 1<\ell<i$. Hence, the edge $\left\{v_{1}, v_{i}\right\}$ is only crossed by edges incident to $v_{n}$. Moreover, any edge that is crossed by $\left\{v_{i}, v_{1}\right\}$ is already crossed by two edges incident to $v_{1}$. Since $G$ is maximal outer-fan-planar, it must contain edge $\left\{v_{i}, v_{1}\right\}$. A similar argument holds for $\left\{v_{n}, v_{i+1}\right\}$.

Lemma 5. Let $G$ be a 3-connected graph with a maximal outer-fan-planar drawing and assume that the drawing contains at least one long edge. Then, $G$ contains a $K_{4}$ with all four vertices drawn consecutively on the circle.

Proof. First consider the case where the graph contains at least two crossing long edges and, thus, by Lemma 3 a scissor. Removing the vertices of a scissor, splits $G$ into two connected components. Assume that we have chosen the scissor such that the smaller of the two components is as small as possible (thus, scissor-free) and that the vertices around $\mathcal{C}$ are labeled such that this scissor is $\left\{v_{1}, v_{i+1}\right\},\left\{v_{i}, v_{n}\right\}$ with $i \leq n-i$, i.e., the component induced by $v_{2}, \ldots, v_{i-1}$ is the smaller one. Recall that by Lemma 4 a scissor induces a $K_{4}$.

If $i=3$, i.e., if $\left\{v_{1}, v_{3}\right\}$ is a 2-hop, then $G$ should contain either $\left\{v_{2}, v_{n}\right\}$ or $\left\{v_{2}, v_{4}\right\}$, as otherwise $v_{1}$ and $v_{3}$ is a separation pair; see Fig. 2d. Say without loss of generality $\left\{v_{2}, v_{n}\right\}$. Then, $v_{1}, v_{2}, v_{3}$ together with $v_{n}$ induce a $K_{4}$ with all vertices consecutive on circle $\mathcal{C}$. 


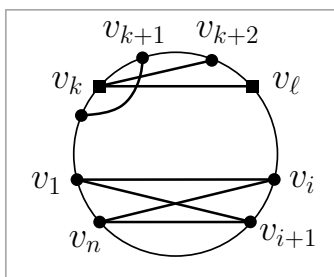

(a)

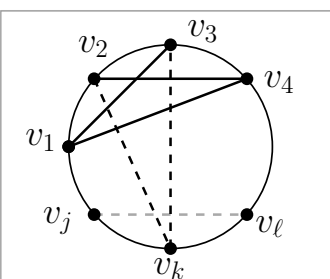

(b)

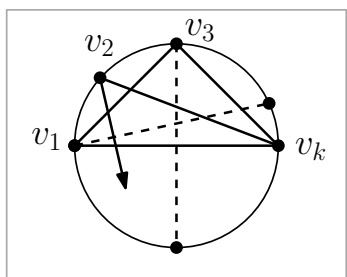

(c)

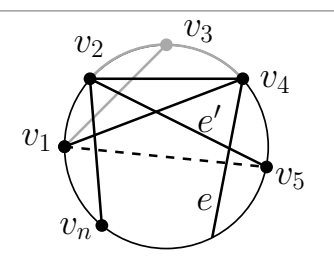

(d)

Fig. 3. Different configurations used in: (a) Lemma 5, (b) Lemma 6, (c) Lemma 7. (d) Lemma 9

If $i>3$, let $\left\{v_{k}, v_{\ell}\right\}, 1 \leq k<\ell \leq i$ be a long edge such that there is no long edge $\left\{v_{k^{\prime}}, v_{\ell^{\prime}}\right\} \neq\left\{v_{k}, v_{\ell}\right\}$ with $k \leq k^{\prime}<\ell^{\prime} \leq \ell$; see Fig. 3a. Then, no long edge is crossing the edge $\left\{v_{k}, v_{\ell}\right\}$, as otherwise by Lemma 3 such a crossing would yield a new scissor, contradicting the choice of $\left\{v_{1}, v_{i+1}\right\}$ and $\left\{v_{i}, v_{n}\right\}$. Since $\left\{v_{k}, v_{\ell}\right\}$ is not crossed by a long edge, it must be crossed by exactly one 2-hop, say $\left\{v_{k-1}, v_{k+1}\right\}$. Now, $\ell-k>3$ is not possible, since we could add the edge $\left\{v_{k+1}, v_{\ell}\right\}$, which is long. Hence, $\ell-k=3$ and by maximality of the outer-fan-planar drawing, $v_{k}, v_{k+1}, v_{k+2}, v_{\ell}$ induces a $K_{4}$ with all vertices consecutive on $\mathcal{C}$. Finally, if $G$ contains no two crossing long edges, let $\left\{v_{k}, v_{\ell}\right\}, 1 \leq k<\ell \leq n$ be a long edge such that there is no long edge $\left\{v_{k^{\prime}}, v_{\ell^{\prime}}\right\} \neq\left\{v_{k}, v_{\ell}\right\}$ with $k \leq k^{\prime}<\ell^{\prime} \leq \ell$. By the same argumentation as above, we obtain that $v_{k}, v_{k+1}, v_{k+2}, v_{\ell}$ induces a $K_{4}$ with all vertices consecutive on $\mathcal{C}$.

Lemma 6. Let $G$ be a 3-connected outer-fan-planar graph with at least six vertices. If $G$ contains a $K_{4}$ with all vertices drawn consecutively on circle $\mathcal{C}$, then this $K_{4}$ contains exactly one vertex of degree three and this vertex is neither the first nor the last of the four vertices.

Proof. Let the vertices around circle $\mathcal{C}$ be labeled so that $v_{1}, v_{2}, v_{3}, v_{4}$ induce a $K_{4}$. Since $v_{1}$ and $v_{4}$ is not a separation pair, there is an edge between $v_{2}$ or $v_{3}$ and a vertex, say $v_{k}$, among $v_{5}, \ldots, v_{n}$. Hence, three out of the four vertices $v_{1}, v_{2}, v_{3}$ and $v_{4}$ have degree at least four; see Fig. 3b. If $v_{3}$ had a neighbor in $v_{5}, \ldots, v_{n}$, then this could only be $v_{k}$, as otherwise $\left\{v_{1}, v_{4}\right\}$ would be crossed by two independent edges. Since $G$ has at least 6 vertices, we assume without loss of generality that $k>5$. Since $v_{4}$ and $v_{k}$ is not a separation pair, there has to be an edge $\left\{v_{\ell}, v_{m}\right\}$ for some $4<\ell<k$ and a $j \notin\{4, \ldots, k\}$. But such an edge would not be possible in an outer-fan-planar drawing.

Lemma 7. Let $G$ be a 3-connected outer-fan-planar graph with at least six vertices. If $G$ contains a $K_{4}$ with a vertex of degree 3 , then this $K_{4}$ has to be drawn consecutively on circle $\mathcal{C}$ in any outer-fan-planar drawing of $G$.

Proof. Observe that any outer-fan-planar drawing of a $K_{4}$ contains exactly one pair of crossing edges. If two 2-hops cross, then all vertices of the $K_{4}$ are consecutive. If the $K_{4}$ contains two crossing long edges, then each of the vertices of the $K_{4}$ is incident to an outer edge not contained in the $K_{4}$; thus, has degree at least four. If a long edge and a 2-hop cross, assume that the vertices around $\mathcal{C}$ are labeled such that $v_{1}, v_{2}, v_{3}, v_{k}$ induce a $K_{4}$ for some $5 \leq k<n$; see Fig. 3c. Since $v_{1}, v_{3}$ and $v_{k}$ are incident to an outer edge not contained in the $K_{4}$, they have degree at least four. We claim that $v_{2}$ has degree at least four. Since $v_{3}$ and $v_{k}$ is not a separation pair, there is an edge between a vertex among $v_{4}, \ldots, v_{k-1}$ and $v_{2}$ or $v_{1}$ and an edge between a vertex among $v_{k+1}, \ldots, v_{n}$ and $v_{2}$ or $v_{3}$. Choosing $v_{1}$ and $v_{3}$ in the first and second case respectively, yields two independent edges crossing $\left\{v_{2}, v_{k}\right\}$. So, $v_{2}$ is connected to a vertex outside $K_{4}$.

Lemma 8. Let $G$ be a 3-connected graph with $n \geq 5$ vertices and let $v \in V[G]$ be a vertex of degree three that is contained in a $K_{4}$. Then, $G-\{v\}$ is 3-connected.

Proof. Let $a, b, c$ and $d$ be four arbitrary vertices of $G-\{v\}$. Since $G$ was 3-connected, there was a path $P$ from $a$ to $b$ in $G-\{c, d\}$. Assume that $P$ contains $v$. Since $v$ is only connected to vertices that are connected to each other, there is also another path from $a$ to $b$ in $G-\{c, d\}$ not containing $v$. Hence, $a$ and $b$ cannot be a separation pair in $G-\{v\}$. Since $a$ and $b$ were arbitrarily selected, $G-\{v\}$ is 3 -connected. 
Lemma 9. Let $G$ be a 3-connected graph with $n>6$ vertices, let $v_{1}, v_{2}, v_{3}$ and $v_{4}$ be four vertices that induce a $K_{4}$, such that the degree of $v_{3}$ is three. Then, $G-\left\{v_{3}\right\}$ has a maximal outer-fan-planar drawing if $G$ has a maximal outer-fan-planar drawing.

Proof. Consider a maximal outer-fan-planar drawing of $G$ on a circle $\mathcal{C}$ and let $v_{1}, v_{2}, v_{3}, v_{4}, \ldots, v_{n}$ be the order of the vertices on $\mathcal{C}$ (recall Lemma 7). Assume to the contrary that after removing $v_{3}$, we could add an edge $e$ to the drawing; see Fig. 3d. By Lemma 6. $\left\{v_{3}, v_{1}\right\}$ is the only edge incident to $v_{3}$ that crosses some edges of $G-\left\{v_{3}\right\}$. Hence, there must be an edge $e^{\prime}$ that is crossed by $e$ and $\left\{v_{3}, v_{1}\right\}$. Since $\left\{v_{3}, v_{1}\right\}$ crosses only edges incident to $v_{2}$ that also cross $\left\{v_{1}, v_{4}\right\}$, it follows that $e^{\prime}$ has to be incident to $v_{2}$. Further, since $G-\left\{v_{3}\right\}$ plus $e$ is outer-fan-planar it follows that $e$ is incident to $v_{1}$ or $v_{4}$. Moreover, since $G$ plus $e$ is not outer-fan-planar it follows that $e$ is incident to $v_{4}$.

Let $i$ be maximal so that there is an edge $\left\{v_{2}, v_{i}\right\}$. If $i \neq n$, then $v_{1}$ and $v_{i}$ is a separation pair: Any edge connecting $\left\{v_{i+1}, \ldots, v_{n-1}\right\}$ to $\left\{v_{2}, v_{3}, \ldots, v_{i-1}\right\}$ and not being incident to $v_{2}$ crosses $\left\{v_{2}, v_{i}\right\}$. But edges crossing $\left\{v_{2}, v_{i}\right\}$ can only be incident to $v_{1}$, a contradiction. Now, let $j>4$ be minimum such that there is an edge $\left\{v_{2}, v_{j}\right\}$. We claim that $j=5$. If this is not the case, then similarly to the previous case $v_{4}$ and $v_{j}$ would be a separation pair in $G-\left\{v_{3}\right\}$ plus $e$, which is not possible due to Lemma 8 .

It follows that $G$ has to contain edge $\left\{v_{1}, v_{5}\right\}$ : Since $G$ is outer-fan-planar, in $G$ there cannot be an edge $\left\{v_{4}, v_{k}\right\}$ for some $k=6, \ldots, n$, since it would cross $\left\{v_{2}, v_{5}\right\}$ which is crossed by $\left\{v_{3}, v_{1}\right\}$. So, $\left\{v_{1}, v_{5}\right\}$ crosses only edges incident to $v_{2}$ that are already crossed by $\left\{v_{3}, v_{1}\right\}$ and $\left\{v_{4}, v_{1}\right\}$. Hence, $\left\{v_{1}, v_{5}\right\}$ could be added to $G$ without violating outer-fan-planarity; a clear contradiction. Since $e$ and $\left\{v_{2}, v_{n}\right\}$ both cross $\left\{v_{1}, v_{5}\right\}$ it follows that $e=\left\{v_{4}, v_{n}\right\}$. But now, $v_{5}$ and $v_{n}$ has to be a separation pair.

Remark 1. Let $G$ be a graph with 6 vertices containing a vertex $v$ of degree three. Then, $G$ is maximal outer-fan-planar if and only if $G-\{v\}$ is a $K_{5}$ missing one of the edges that connects a neighbor of $v$ to one of the other two vertices.

Lemma 10. It can be tested in linear time whether a graph is a complete 2-hop graph. Moreover, if a graph is a complete 2-hop graph, then it has a constant number of outer-fan-planar embeddings and these can be constructed in linear time.

Proof. Let $G$ be an $n$-vertex graph. We test whether $G$ is a complete 2 -hop as follows. If $n \in\{4,5\}$, then $G$ is either $K_{4}$ or $K_{5}$. Otherwise, check first whether all vertices have degree four. If so, pick one vertex as $v_{1}$, choose a neighbor as $v_{2}$ and a common neighbor of $v_{1}$ and $v_{2}$ as $v_{3}$ (if no such common neighbor exists, then $G$ is not a complete 2 -hop). Assume now that we have already fixed $v_{1}, \ldots, v_{i}, 3 \leq i<n$. Test whether there is a unique vertex $v \in V \backslash\left\{v_{1}, \ldots, v_{i}\right\}$ that is adjacent to $v_{i}$ and $v_{i-1}$. If so, set $v_{i+1}=v$. Otherwise reject. If we have fixed the order of all vertices check whether there are only outer edges and 2-hops. Do this for any possible choices of $v_{2}$ and $v_{3}$, i.e., for totally at most 6 choices.

Remark 2. No degree 3 vertex can be added to an $n$-vertex complete 2-hop with $n \geq 5$.

We are now ready to describe our algorithm. If the graph is not a complete 2-hop graph, recursively try to remove a vertex of degree 3 which is contained in a $K_{4}$. If $G$ is maximal outer-fan-planar, Lemmas 5 and 6 guarantee that such a vertex always exists in the beginning. Remark 2 guarantees that also in subsequent steps there is a long edge and, thus, Lemmas 8 and 9 guarantee that also in subsequent steps, we can apply Lemma 5 as long as we have at least six vertices. Remark 1 guarantees that we can also remove two more vertices of degree 3 ending with a triangle.

At this stage, we already know that if the graph is outer-fan-planar, it is indeed maximal outer-fan-planar. Either, we started with a complete 2-hop graph or we iteratively removed vertices of degree three yielding a triangle. Note that in the latter case we must have started with $3 n-6$ edges. On the other hand, if we apply the above procedure to an $n$-vertex 3 -connected maximal outer-fan-planar graph, we get that the number of edges is exactly $2 n$ or $3 n-6$.

Finally, we try to reinsert the vertices in the reversed order in which we have deleted them. By Lemma 7 we can insert the vertex of degree three only between its neighbor, i.e., there are at most two possibilities where we could insert the vertex. Lemma 11 guarantees that in total, we have to check at most four possible drawings for $G$. A summary of our approach is also given in Algorithm 1. 


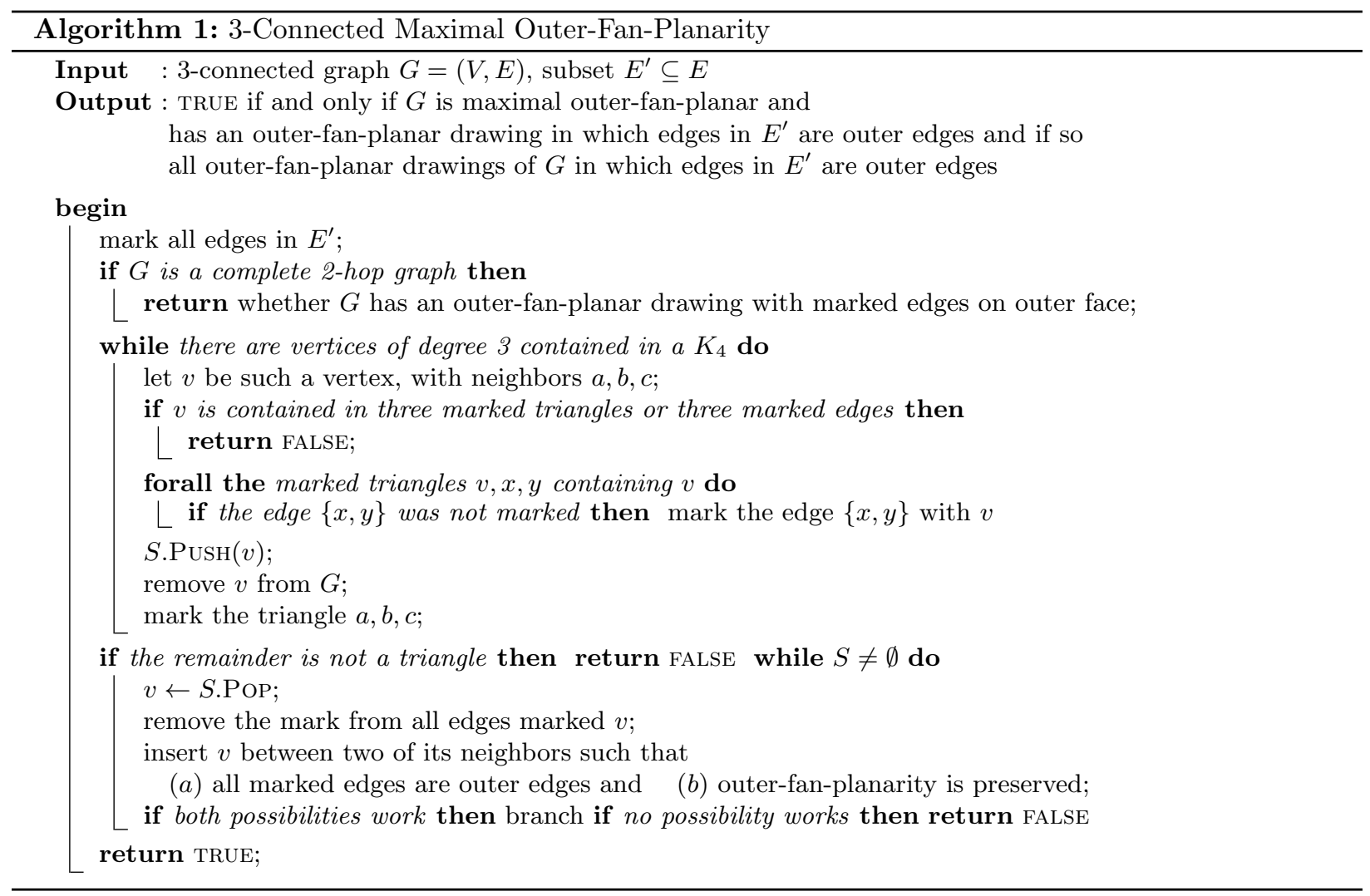


Lemma 11. When reinserting a sequence of degree 3 vertices starting from a triangle, at most the first two vertices have two choices where they could be inserted.

Proof. Let $H$ be a outer-fan-planar graph and let three consecutive vertices $v_{1}, v_{2}, v_{3}$ induce a triangle. Assume, we want to insert a vertex $v$ adjacent to $v_{1}, v_{2}, v_{3}$. By Lemma 6, we have to insert $v$ between $v_{1}$ and $v_{2}$ or between $v_{2}$ and $v_{3}$. Note that the edges that are incident to $v_{2}$ and cross $\left\{v_{1}, v_{3}\right\}$ are also crossed by an edge $e$ incident to $v$. So, if there is an edge incident to $v_{2}$ that was already crossed twice before inserting $v$, this would uniquely determine whether $e$ is incident to $v_{1}$ or $v_{3}$ and, thus, where to insert $v$.

We will now show that after the first insertion each relevant vertex is incident to an edge that is crossed at least twice. When we insert the first vertex we create a $K_{4}$. From the second vertex on, whenever we insert a new vertex, it is incident to an edge that is crossed at least twice. Also, after inserting the second degree 3 vertex, three among the four vertices of the initial $K_{4}$ are also incident to an edge that is crossed at least twice. The forth vertex of the initial $K_{4}$ is not the middle vertex of a triangle consisting of three consecutive vertices. It can only become such a vertex if its incident inner edges are crossed by a 2-hop. But then these inner edges are all crossed at least twice.

Summarizing, we obtain the following theorem; in order to exploit this result in the biconnected case, it is also tested whether a prescribed subset (possibly empty) of edges can be drawn as outer edges.

Theorem 1. Given a 3-connected graph $G$ with a subset $E^{\prime}$ of its edge set, it can be tested in linear time whether $G$ is maximal outer-fan-planar and has an outer-fan-planar drawing such that the edges in $E^{\prime}$ are outer edges. Moreover if such a drawing exists, it can be constructed in linear time.

Proof. Let $n$ be the number of vertices. By Lemma 10, a complete 2-hop graph has only a constant number of outer-fan-planar embeddings which can be computed in linear time. In the other case, any vertex that was removed from the queue will never be appended again. Hence, there are at most $n$ iterations in the first part of Algorithm 1 .

To check whether the degree three vertices can be reinserted back in the graph, we only have to consider in total four different embeddings. Assume that we want to insert a vertex $v$ into an outer triangle $v_{1}, v_{2}, v_{3}$. Then we just have to check whether $v_{1}$ or $v_{3}$ are incident to edges other than the edge $\left\{v_{1}, v_{3}\right\}$ that cross an edge incident to $v_{2}$. This can be done in constant time by checking only two pairs of edges.

\subsection{The Biconnected Case}

We now show how to test outer-fan-planar maximality on a biconnected graph.

Lemma 12. Let $v_{1}, \ldots, v_{n}$ be the order of the vertices around the circle in an outer-fan-planar drawing of a 3 -connected graph $G$. If we can add a vertex $v$ between $v_{1}$ and $v_{n}$ with an edge $\left\{v, v_{i}\right\}$ for some $i=2, \ldots, n-1$, then $i=2$ or $i=n-1$.

Proof. Otherwise, since $v_{1}, v_{i}$ cannot be a separation pair of $G$, there has to be an edge from a $v_{k}$ for some $k=2, \ldots, i-1$ that crosses $\left\{v, v_{i}\right\}$ and hence an edge $\left\{v_{k}, v_{n}\right\}$. Since $v_{n}, v_{i}$ cannot be a separation pair of $G$, there has to be an edge $\left\{v_{1}, v_{\ell}\right\}$ for some $\ell=i+1, \ldots, n-1$. But now there are three independent edges crossing.

We say that an outer edge $\left\{v_{1}, v_{n}\right\}$ is porous around $v_{1}$ if we could add a vertex $v$ between $v_{1}$ and $v_{n}$ and an edge $\left\{v, v_{2}\right\}$ maintaining outer-fan-planarity. Note that any edge of a simple cycle, i.e., of the skeleton of an $S$-node is porous around any of its end-vertices. Any outer edge of a $K_{4}$ is porous around any of its end-vertices; see Fig. 4.

We use the SPQR-tree of a biconnected graph to characterize whether it is maximal outer-fan-planar.

Theorem 2. A biconnected graph is maximal outer-fan-planar iff the following hold:

1) The skeleton of any $R$-node is maximal outer-fan-planar and has an outer-fan-planar drawing in which all virtual edges are outer edges, 


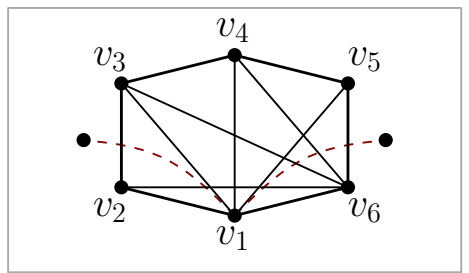

(a)

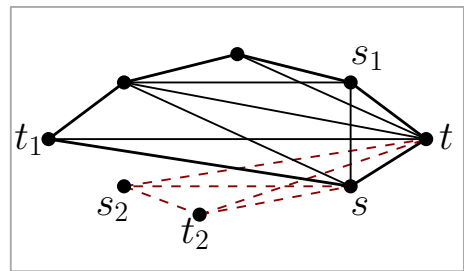

(b)

Fig. 4. (a) In the solid graph, edge $\left\{v_{2}, v_{3}\right\}\left(\left\{v_{5}, v_{6}\right\}\right)$ is porous around $v_{2}$ ( $v_{6}$, resp.). (b) Illustration of Case $5 \mathrm{~b}$ of Theorem 2 .

2) No $R$-node is adjacent to an $R$-node or an $S$-node,

3) All $S$-nodes have degree three,

4) All $P$-nodes have degree three and are adjacent to a $Q$-node, and

5) Let $G_{1}$ and $G_{2}$ be the skeleton of the two neighbors of a $P$-node other than the $Q$-node and let $\{s, t\}$ be the common virtual edge of $G_{1}$ and $G_{2}$. Then, $G_{i}, i=1,2$ must not admit an outer-fan-planar drawing with $t_{i}, s, t, s_{i}$ being consecutive around the circle and

(a) edge $\{s, t\}$ is porous in both $G_{1}$ and $G_{2}$ around the same vertex, or

(b) edge $\left\{t_{1}, s\right\}\left(\left\{s_{2}, t\right\}\right)$ is real and porous around $s$ ( $t$, resp.), or

(c) edge $\left\{s_{1}, t\right\}\left(\left\{t_{2}, s\right\}\right)$ is real and porous around $t$ ( $s$, resp.).

Proof. Let $G$ be a biconnected graph.

$\Leftarrow$ : Clearly, if 1 and 4 are fulfilled, then $G$ is outer-fan-planar. Just merge skeletons at common virtual edges such that one skeleton is in the outer face of the other skeleton. It remains to show maximality.

The skeleton of each node is maximal outer-fan-planar. Assume now that we have already merged some nodes of the SPQR-tree obtaining a maximal outer-fan-planar graph $H$ and that we next want to merge $H$ with a skeleton $G_{x}$ at a virtual edge $\{s, t\}$ obtaining a graph $H^{\prime}$. There is nothing to show if $G_{x}$ is the skeleton of a $P$-node. So assume that $G_{x}$ is a triangle or a 3 -connected graph. Consider a fixed outer-fan-planar drawing of $H^{\prime}$.

We first show that the vertices of $G_{x}$ are consecutive on the circle. If $G_{x}$ is a triangle, this follows directly from Lemma 12. Condition 5b, and its symmetric counter part. Assume now that $G_{x}$ is 3-connected. Note that the outer-fan-planar drawing of $H^{\prime}$ also induce outer-fan-planar drawings of $G_{x}$ and $H$. By maximality these two drawings contain all outer edges. Let now $e$ be an edge that was an outer edge in one of the two subgraphs - say $G_{x}$ - but not incident to $s$ or $t$. Then, $e$ can only be an outer edge or a 2-hop in $H^{\prime}$ : if $e^{\prime}$ is not an outer edge in $H$, it crosses at least two outer edges of the other subgraph - here $H$. Hence, starting from $s$ and $t$ the vertices must be ordered as follows around the circle: first there might be one or more vertices of one of the two subgraphs $G_{x}$ or $H$. Then, there might be alternatingly a vertex from $H$ and $G_{x}$, finally there could be again several vertices from one of the two subgraphs $G_{x}$ or $H$. Using that $G_{x}$ is 3-connected and a case distinction on whether the two sequences of vertices next to $s$ and $t$, respectively, are chosen from $H$ or $G_{x}$, respectively, we obtain that the alternating part on the circle has to be empty.

Hence, $G_{x}$ has to be inserted next to $s$ or $t$. Now, Condition $5 \mathrm{~b}$ implies that $G_{x}$ must be inserted right between $s$ and $t$. Hence, the only edge that could be inserted into the drawing would be an edge crossing $\{s, t\}$ which is prohibited by Condition $5 \mathrm{~b}$ and its symmetric counter part.

$\Rightarrow$ : Assume again that we have already merged some nodes of the SPQR-tree obtaining a maximal outerfan-planar graph $H$ and that we next want to merge $H$ with a skeleton $G_{x}$ at a virtual edge $\{s, t\}$ obtaining a graph $H^{\prime}$. Note that in a maximal outer-fan-planar drawing all outer edges have to be present. This implies especially, that the vertices of $G_{x}$ have to be consecutive in an outer-fan-planar drawing of $H^{\prime}$ with $s$ and $t$ being the first and the last vertex and that we cannot draw the skeletons of two nodes adjacent to one $P$-node on the same side of the respective virtual edge. Otherwise, we obtain 
the situation indicated in Fig. 4b This implies 1, 5b, and its symmetric counterpart. Moreover, all virtual edges have to be real edges which implies 2 and, combined with the previous observation, also 4 If the skeleton of an $S$-node would be a cycle of length greater than three, we could add chords, contradicting maximality. Hence, 3 is fulfilled. Finally, when combining two components in parallel, we should not be able to add an edge from one component to the other routed over the virtual edge. This implies 5 a.

\section{The NP-hardness of the Fan-Planarity with Fixed Rotation System Problem}

In this section, we study the FAn-Planarity with Fixed Rotation System problem (FP-FRS), that is, the problem of deciding whether a graph $G=(V, E)$ with a fixed rotation system $\mathcal{R}$ admits a fan-planar drawing preserving $\mathcal{R}$.

\section{Theorem 3. Fan-Planarity with Fixed Rotation System is NP-hard.}

Proof. We prove the statement by using a reduction from 3-PARTition (3P). An instance of 3P is a multiset $A=\left\{a_{1}, a_{2}, \ldots, a_{3 m}\right\}$ of $3 m$ positive integers in the range $(B / 4, B / 2)$, where $B$ is an integer such that $\sum_{i=1}^{3 m} a_{i}=m B$. 3P asks whether $A$ can be partitioned into $m$ subsets $A_{1}, A_{2}, \ldots, A_{m}$, each of cardinality 3 , such that the sum of the numbers in each subset is $B$. As 3P is strongly NP-hard [16, it is not restrictive to assume that $B$ is bounded by a polynomial in $m$.

Given an instance $A$ of $3 \mathrm{P}$, we show how to transform it into an instance $\left\langle G_{A}, \mathcal{R}_{A}\right\rangle$ of FP-FRS, by a polynomial-time transformation, in such a way that the former is a Yes-instance of $3 \mathrm{P}$ if and only if the latter is a Yes-instance of FP-FRS.

Before describing our transformation in detail, we need to introduce the concept of barrier gadget. An $n$-vertex barrier gadget is a graph consisting of a cycle of $n \geq 5$ vertices plus all its 2-hop edges; a barrier gadget is therefore a maximal outer-2-planar graph. We make use of barrier gadgets in order to constraint the routes of some specific paths of $G_{A}$, as will be clarified soon. We exploit the following property of barrier gadgets. Let $G$ be a biconnected fan-planar graph containing a barrier subgraph $G_{b}$, and let $\Gamma$ be a fan-planar drawing of $G$ such that drawing $\Gamma_{b}$ of $G_{b}$ in $\Gamma$ is maximal outer-2-planar. Then, no path $\pi$ of $G-G_{b}$ can enter inside the boundary cycle of $\Gamma_{b}$ and cross a 2-hop edge. Indeed, every 2-hop edge $e_{b}$ of $\Gamma_{b}$ is crossed by two other 2-hop edges having an end-vertex in common, hence if $e_{b}$ were crossed by $\pi$, then $e_{b}$ would be crossed by two independent edges. On the other hand, if path $\pi$ enters inside $\Gamma_{b}$ without crossing any 2-hop edge, then it must cross twice a same boundary edge $e_{b}^{\prime}$ because of the biconnectivity of $G$; namely, if path $\pi$ enters in $\Gamma_{b}$, then it must also exit from it passing through the same boundary edge. In this case, the only possibility that preserves the fan-planarity of $\Gamma$ is that $\pi$ crosses $e_{b}^{\prime}$ with two consecutive edges, thus forming a fan-crossing. Otherwise, $e_{b}^{\prime}$ would be crossed either by two independent edges of $\pi$ or by a same edge of $\pi$ twice, but both these cases are not allowed in a (simple) fan-planar drawing.

Now, we are ready to describe how to transform an instance $A$ of $3 \mathrm{P}$ into an instance $\left\langle G_{A}, \mathcal{R}_{A}\right\rangle$ of FPFRS. We start from the construction of graph $G_{A}$ which will be always biconnected. First of all, we create a global ring barrier by attaching four barrier gadgets $G_{t}, G_{r}, G_{b}$ and $G_{l}$ as depicted in Figure 5 . $G_{t}$ is called the top beam and contains exactly $3 m K$ vertices, where $K=\lceil B / 2\rceil+1 . G_{r}$ is the right wall and has only five vertices. $G_{b}$ and $G_{r}$ are called the bottom beam and the left wall, respectively, and they are defined in a specular way. Observe that $G_{t}, G_{r}, G_{b}$ and $G_{l}$ can be embedded so that all their vertices are linkable to points within the closed region delimited by the global ring barrier. Then, we connect the top and bottom beams by a set of $3 m$ columns, see Figure 5 for an illustration of the case $m=3$. Each column consists of a stack of $2 m-1$ cells; a cell consists of a set of pairwise disjoint edges, called the vertical edges of that cell. In particular, there are $m-1$ bottommost cells, one central cell and $m-1$ topmost cells. Cells of a same column are separated by $2 m-2$ barrier gadgets, called floors. Central cells (that are $3 m$ in total) have a number of vertical edges depending on the elements of $A$. Precisely, the central cell $C_{i}$ of the $i$-th column contains $a_{i}$ vertical edges connecting its delimiting floors $(i \in\{1,2, \ldots, 3 m\})$. Instead, all the remaining cells have, each one, $K$ vertical edges. Hence, a non-central cell contains more edges than any central cell. Further, the number of vertices of a floor is given by the number of its incident vertical edges minus two. Let $u$ and $v$ be the "central" vertices of the left and right walls, respectively (see also Figure 5). We conclude the 


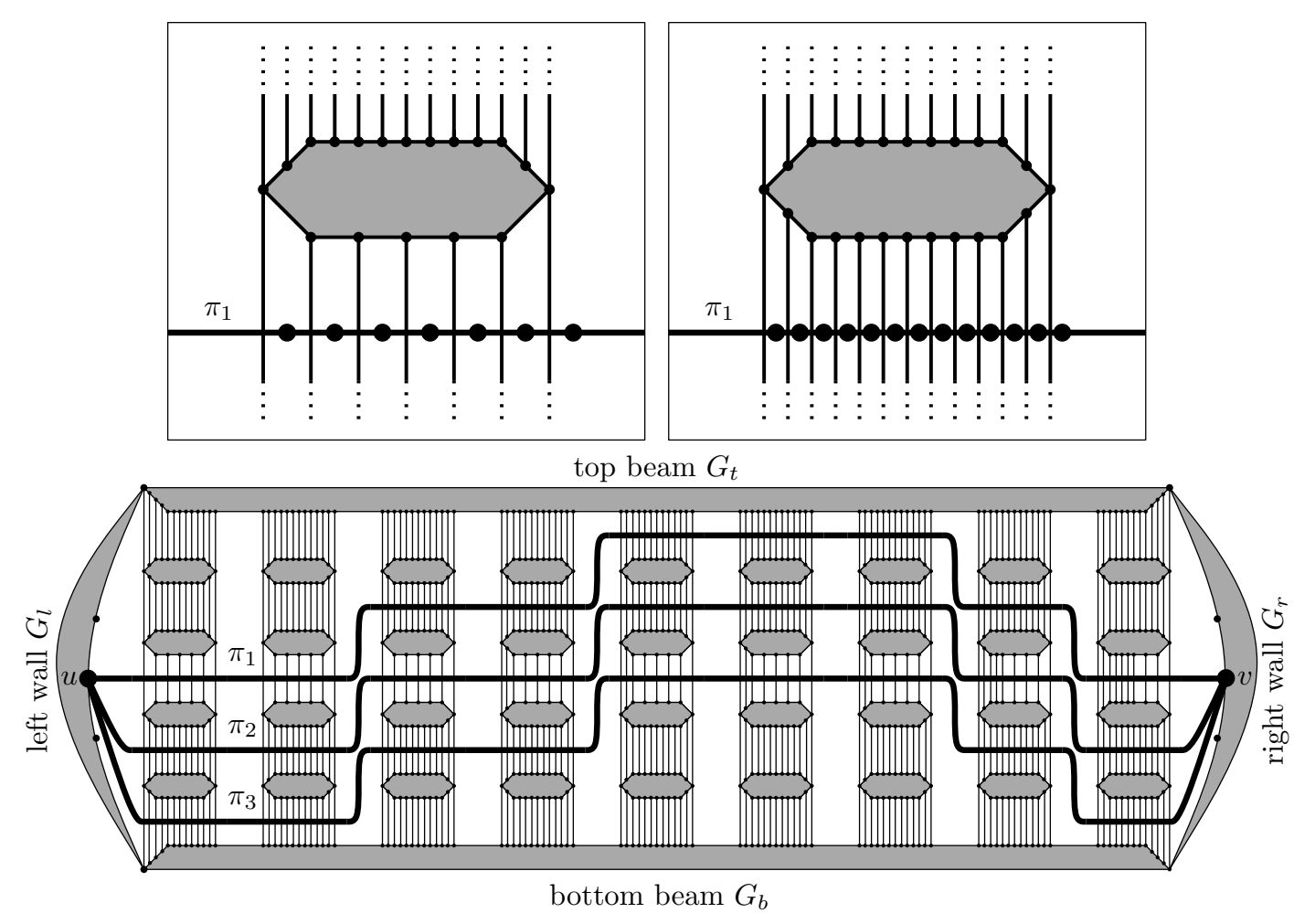

Fig. 5. Illustration of the reduction of FP-FRS from $3 \mathrm{P}$, where $m=3, A=\{7,7,7,8,8,8,8,9,10\}$ and $B=24$. Transversal paths are routed according to the following solution of $3 \mathrm{P}: A_{1}=\{7,7,10\}, A_{2}=\{7,8,9\}$ and $A_{3}=$ $\{8,8,8\}$. The top-left and top-right boxes show a zoom of the first central cell and of the non-central cell of the 3-rd column traversed by path $\pi_{1}$, respectively.

construction of graph $G_{A}$ by connecting vertices $u$ and $v$ with $m$ pairwise internally disjoint paths, called the transversal paths of $G_{A}$; each transversal path has exactly $(3 m-3) K+B$ edges.

Concerning the choice of a rotation system $\mathcal{R}_{A}$, we define a cyclic ordering of edges around each vertex that is compatible with the following constraints: (i) every barrier gadget can be embedded with all its 2-hop edges inside its boundary cycle; (ii) the global ring barrier can be embedded with only four vertices on the outer face; (iii) columns can be embedded inside the region delimited by the global ring barrier without crossing each other; (iv) vertical edges of cells can be embedded without creating crossings; (v) transversal paths are attached to the left and right walls such that the ordering of their edges around $u$ is specular to the ordering around $v$; this choice makes it possible to avoid crossings between any two transversal paths. From what said, it is straightforward to see that an instance of $3 \mathrm{P}$ can be transformed into an instance of FP-FRS in polynomial time in $m$.

We now prove that a Yes-instance of $3 \mathrm{P}$ is transformed into a Yes-instance of FP-FRS, and vice-versa. Let $A$ be a $Y e s$-instance of $3 \mathrm{P}$, we show that $\left\langle G_{A}, \mathcal{R}_{A}\right\rangle$ admits a fan-planar drawing $\Gamma_{A}$ preserving $\mathcal{R}_{\mathcal{A}}$. We preliminarily observe that such a drawing is easy to compute if one omits all the transversal paths. It is essentially a drawing like that one depicted in Figure 5, where columns are one next to the other within the closed region delimited by the global ring barrier. However, by exploiting a solution $\left\{A_{1}, A_{2}, \ldots, A_{m}\right\}$ of $3 \mathrm{P}$ for the instance $A$, also the transversal paths can be easily embedded without violating the fan-planarity. The idea is to route these paths in such a way that: (R.1) they do not cross each other; (R.2) they do not cross any barrier; (R.3) each path passes through exactly 3 central cells and $3 m-3$ non-central cells; and (R.4) each cell is traversed by at most one path. More precisely, each transversal path $\pi_{j}$ is biunivocally associated with a subset $A_{j}(j \in\{1,2, \ldots, m\})$ and the three central cells it passes through have three sets of vertical edges whose cardinalities form a triple of integers matching the three integers of $A_{j}$. Paths 
are routed by sweeping columns from left to right and by proceeding as follows. Let $C_{1}$ be the 1-st central cell; $C_{1}$ has $a_{1}$ vertical edges by construction. The transversal path passing through $C_{1}$ is a path $\pi_{j(1)}$ such that subset $A_{j(1)}$ contains an integer equal to $a_{1}$. The remaining transversal paths are routed until the 1-st column by preserving the cyclic edge-ordering around $u$ and by respecting conditions R.1, R.2, R.3 and R.4; note that condition R.3 cannot be violated at this point. Suppose now that all paths have been already routed until the $(i-1)$-th column, for some $i \geq 2$, and suppose also that conditions R.k $(1 \leq k \leq 4)$ are satisfied. Then, there is at least a path $\pi_{j(i)}$ whose corresponding subset $A_{j(i)}$ contains an integer $a_{i}$ that has not yet been considered. Path $\pi_{j(i)}$ is the next path that goes through the central cell $C_{i}$. The remaining paths are routed in such a way that their "vertical distances" to path $\pi_{j(i)}$, in terms of number of cells, are unchanged when passing from the $(i-1)$-th column to the $i$-th column. Eventually, each transversal path crosses exactly $(3 m-3) K+B$ vertical edges, which is the same number of its edges. Therefore, it is possible to draw these paths by ensuring that each of their edges crosses exactly one vertical edge, which preserves the fan-planarity. Hence, eventually we get a fan-planar drawing $\Gamma_{A}$ preserving the rotation system $\mathcal{R}_{A}$.

We conclude the proof by showing that if $\left\langle G_{A}, \mathcal{R}_{A}\right\rangle$ is a Yes-instance of FP-FRS, then $A$ is a Yes-instance of 3P. Let $\Gamma_{A}$ be a fan-planar drawing of $G_{A}$ preserving the rotation system $\mathcal{R}_{A}$. We first observe that the top beam and the bottom beam are disjoint, otherwise there would be at least a 2-hope edge in one beam that is crossed by another edge of the other beam, thus violating the fan-planarity. We also note that columns can partially cross each other, but this does not actually affect the validity of the proof. Indeed, an edge $e$ of a column $L$ might cross an edge $e^{\prime}$ of another column $L^{\prime}$ only if $e$ is incident to a vertex in the rightmost (leftmost) side of $L, e^{\prime}$ is a leftmost (rightmost) vertical edge of $L^{\prime}$, and $L$ and $L^{\prime}$ are two consecutive columns. With a similar argument, it is immediate to see that vertices $u$ and $v$ must be separated by all the columns. Therefore, every transversal path satisfies conditions R.1, R.2 and it must pass through at least three central cells, if not it would cross a number of pairwise disjoint edges that is greater than the number of its edges, hence $\Gamma_{A}$ would not be fan-planar. On the other hand, because of condition R.4, which is obviously satisfied, there cannot be any transversal path passing through more than three central cells. Otherwise, there would be some other transversal path that traverses a number of central cells that is strictly less than three. Hence, also condition R.3 is satisfied. In conclusion, every transversal path $\pi_{j}(j \in\{1,2, \ldots, m\})$ crosses $(3 m-3) K+B$ vertical edges and traverses exactly three central cells $C_{1 j}, C_{2 j}$ and $C_{3 j}$. If $m\left(C_{1 j}\right), m\left(C_{2 j}\right)$ and $m\left(C_{3 j}\right)$ denote the number of edges of these cells, then $m\left(C_{1 j}\right)+m\left(C_{2 j}\right)+m\left(C_{3 j}\right)=B$, because each non-central cell has $K$ edges. Therefore, the partitioning of $A$ defined by $A_{1}, A_{2}, \ldots, A_{m}$, where $A_{j}=\left\{m\left(C_{1 j}\right), m\left(C_{2 j}\right), m\left(C_{3 j}\right)\right\}$, is a solution of $3 \mathrm{P}$ for the instance $A$.

\section{References}

1. Ackerman, E.: On the maximum number of edges in topological graphs with no four pairwise crossing edges. Discrete \& Computational Geometry 41(3), 365-375 (2009)

2. Agarwal, P.K., Aronov, B., Pach, J., Pollack, R., Sharir, M.: Quasi-planar graphs have a linear number of edges. Combinatorica 17(1), 1-9 (1997)

3. Argyriou, E.N., Bekos, M.A., Symvonis, A.: The straight-line RAC drawing problem is np-hard. J. Graph Algorithms Appl. 16(2), 569-597 (2012)

4. Auer, C., Bachmaier, C., Brandenburg, F.J., Gleißner, A., Hanauer, K., Neuwirth, D., Reislhuber, J.: Recognizing outer 1-planar graphs in linear time. In: Wismath, S., Wolff, A. (eds.) GD 2013. LNCS, vol. 8242, pp. 107-118. Springer, Heidelberg (2013)

5. Bodendiek, R., Schumacher, H., Wagner, K.: Über 1-optimale graphen. Mathematische Nachrichten 117(1), 323339 (1984)

6. Cabello, S., Mohar, B.: Adding one edge to planar graphs makes crossing number and 1-planarity hard. CoRR abs/1203.5944 (2012)

7. Cheong, O., Har-Peled, S., Kim, H., Kim, H.S.: On the number of edges of fan-crossing free graphs. In: Cai, L., Cheng, S.W., Lam, T.W. (eds.) ISAAC 2013. LNCS, vol. 8283, pp. 163-173. Springer, Heidelberg (2013)

8. Dehkordi, H.R., Eades, P.: Every outer-1-plane graph has a right angle crossing drawing. Int. J. Comput. Geometry Appl. 22(6), 543-558 (2012)

9. Didimo, W., Eades, P., Liotta, G.: Drawing graphs with right angle crossings. Theor. Comput. Sci. 412(39), $5156-5166(2011)$ 
10. Eades, P., Hong, S.H., Katoh, N., Liotta, G., Schweitzer, P., Suzuki, Y.: A linear time algorithm for testing maximal 1-planarity of graphs with a rotation system. Theor. Comput. Sci. 513, 65-76 (2013)

11. Eades, P., Liotta, G.: Right angle crossing graphs and 1-planarity. Discrete Applied Mathematics 161(7-8), 961969 (2013)

12. Eggleton, R.: Rectilinear drawings of graphs. Utilitas Mathematica 29, 149 - 172 (1986)

13. Fabrici, I., Madaras, T.: The structure of 1-planar graphs. Discrete Mathematics 307(7-8), 854-865 (2007)

14. Fáry, I.: On straight line representations of planar graphs. Acta Sci. Math. Szeged 11, $229-233$ (1948)

15. Fox, J., Pach, J., Suk, A.: The number of edges in k-quasi-planar graphs. SIAM J. Discrete Math. 27(1), 550-561 (2013)

16. Garey, M.R., Johnson, D.S.: Computers and Intractability: A Guide to the Theory of NP-Completeness. W. H. Freeman \& Co., New York, NY, USA (1979)

17. Grigoriev, A., Bodlaender, H.L.: Algorithms for graphs embeddable with few crossings per edge. Algorithmica 49(1), 1-11 (2007)

18. Gutwenger, C., Mutzel, P.: A linear time implementation of SPQR-trees. In: Marks, J. (ed.) GD 2000. LNCS, vol. 1984, pp. 77-90. Springer, Heidelberg (2001)

19. Hong, S.H., Eades, P., Katoh, N., Liotta, G., Schweitzer, P., Suzuki, Y.: A linear-time algorithm for testing outer-1-planarity. In: Wismath, S., Wolff, A. (eds.) GD 2013. LNCS, vol. 8242, pp. 71-82. Springer, Heidelberg (2013)

20. Hong, S.H., Eades, P., Liotta, G., Poon, S.H.: Fáry's theorem for 1-planar graphs. In: Gudmundsson, J., Mestre, J., Viglas, T. (eds.) COCOON. LNCS, vol. 7434, pp. 335-346. Springer, Heidelberg (2012)

21. Kaufmann, M., Ueckerdt, T.: The density of fan-planar graphs. CoRR abs/1403.6184 (2014)

22. Korzhik, V.P., Mohar, B.: Minimal obstructions for 1-immersions and hardness of 1-planarity testing. Journal of Graph Theory 72(1), 30-71 (2013)

23. Nagamochi, H.: Straight-line drawability of embedded graphs. Technical Reports 2013-005, Department of Applied Mathematics and Physics, Kyoto University (2013)

24. Pach, J., Radoicic, R., Tóth, G.: Relaxing planarity for topological graphs. In: Akiyama, J., Kano, M. (eds.) JCDCG 2002. LNCS, vol. 2866, pp. 221-232. Springer, Heidelberg (2002)

25. Pach, J., Tóth, G.: Graphs drawn with few crossings per edge. Combinatorica 17(3), 427-439 (1997)

26. Purchase, H.C.: Effective information visualisation: a study of graph drawing aesthetics and algorithms. Interacting with Computers 13(2), 147-162 (2000)

27. Ringel, G.: Ein Sechsfarbenproblem auf der Kugel. Abh. Math. Sem. Univ. Hamburg 29, $107-117$ (1965)

28. Thomassen, C.: Rectilinear drawings of graphs. Journal of Graph Theory 12(3), 335-341 (1988) 